\title{
The Karush-Kuhn-Tucker optimality conditions in minimum weight design of elastic rotating disks with variable thickness and density
}

\author{
Sanaz Jafari
}

Department of Mechanical Engineering, Babol University of Technology, P.O.Box 484, Babol, Iran

\begin{tabular}{|c|c|}
\hline A R T I C L E I N F O & A B S T R A C T \\
\hline $\begin{array}{l}\text { Article history: } \\
\text { Received } 1 \text { May } 2010 \\
\text { Received in revised form } \\
\text { June, } 20,2011 \\
\text { Accepted } 21 \text { June } 2011 \\
\text { Available online } \\
\text { 22 June 2011 } \\
\text { Keywords: } \\
\text { Rotating annular disk } \\
\text { Variable thickness } \\
\text { Variable density }\end{array}$ & $\begin{array}{l}\text { Rotating discs work mostly at high angular velocity. High speed results in large centrifugal } \\
\text { forces in discs and induces large stresses and deformations. Minimizing weight of such disks } \\
\text { yields various benefits such as low dead weights and lower costs. In order to attain a certain and } \\
\text { reliable analysis, disk with variable thickness and density is considered. Semi-analytical } \\
\text { solutions for the elastic stress distribution in rotating annular disks with uniform and variable } \\
\text { thicknesses and densities are obtained under plane stress assumption by authors in previous } \\
\text { works. The optimum disk profile for minimum weight design is achieved by the Karush-Kuhn- } \\
\text { Tucker (KKT) optimality conditions. Inequality constrain equation is used in optimization to } \\
\text { make sure that maximum von Mises stress is always less than yielding strength of the } \\
\text { material of the disk. }\end{array}$ \\
\hline
\end{tabular}

\section{Introduction}

Rotating discs are historically, areas of research and studies due to their vast utilization in industry such as gears, turbine rotors, flywheels, shrink fits etc. The analytical solutions of rotating solid disks with constant thickness were discussed for elastic-perfectly plastic (Gamer, 1983) and for linearly hardening materials (Gamer, 1984; Gamer, 1985). Güven extended these works to annular disks of variable thickness and variable density (Güven, 1992) and to fully plastic variable thickness solid disks with constant thickness in the central portion (Güven, 1994). Variational iteration solution of elastic non-uniform thickness and density rotating disks by Hojjati and Jafari (Hojjati \& Jafari, 2007) and theoretical and numerical analyses of rotating discs of non-uniform thickness and density at elastic-linear hardening material by variable material property method by Hojjati and Hassani (2008) are some of the newly published researches in this field. Adomian's decomposition and homotopy perturbation methods also have been used by Hojjati and Jafari for the solution of elastic (Hojjati \& Jafari, 2008) and elastic-strain hardening (Hojjati \& Jafari, 2009) non- uniform thickness and density rotating annular disks. Weight usually is attended in design and manufacturing of rotating disk as the major parameter for optimization. Malkov and Salganskaya (Malkov et al., 1976) used numerical methods for optimization of rotating disks, but did not consider nonlinearity of constraints in the

* Corresponding author. Tel: + 981113234201

E-mail: jaafari.sanaz@yahoo.com (S. Jafari)

(C) 2011 Growing Science Ltd. All rights reserved. doi: $10.5267 /$ j.ijiec.2011.06.006 
optimization process. Fox (1970) optimized a rotating disk using the feasible direction method (Vanderplaats, 1990) also used the gradient of constraints. Zienkiewicz and Campbell (Zienkiewicz \& Campbell, 1973) used sequential linear programming methods for optimization of rotating disk. In addition, in a different approach, Chen Cheu (1990) optimized a rotating disk profile by combining the finite element method with the feasible direction method.

The main objective of this work is the utilization of the solutions achieved by the authors in their previous works to minimize the weight of the disk under a certain condition of von-Mises stress be kept under the allowable value. In fact, the focus of this research is to identify those geometry parameters that fulfill these constraints using KKT conditions for optimization. The KKT conditions are discussed in details in (Rao, 2009; Peressini et al., 1988; Wide et al., 1970).

\section{Theoretical Background}

\subsection{Governing equation of rotating disk}

Assuming that stresses do not vary over the thickness of the disk, let $h$ be the thickness of the disk (Fig.1), varying with radius, i.e. $h=h(r)$.

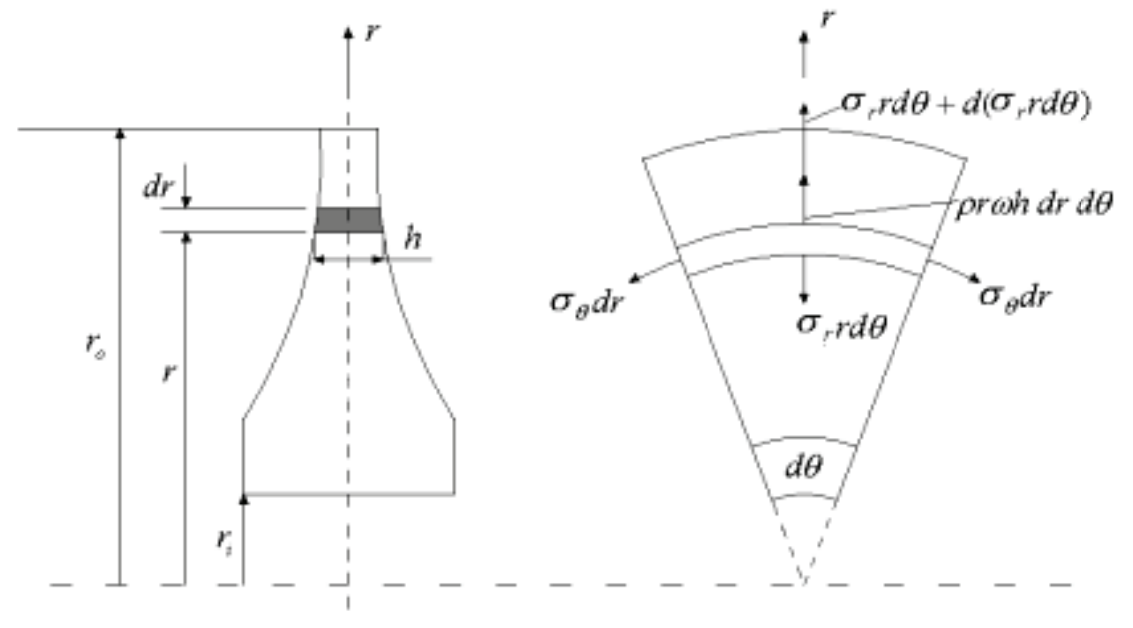

Fig. 1. Disk profile and acting forces on an element

Simplifying the radial equilibrium condition for an infinitesimal element of the disk gives (Hojjati \& Jafari, 2008; Timoshenko, 1970; Güven, 1995):

$\frac{d}{d r}\left(h r \sigma_{r}\right)-h \sigma_{\theta}=-h \rho \omega^{2} r^{2}$,

where $\rho=\rho(r)$ is the distribution function of density, and $\omega$ is the angular velocity of the disk.

In the plane stress $\left(\sigma_{z}=0\right)$ the stress-displacement relations are given by Timoshenko and Goodier (Timoshenko, 1970; Güven, 1995):

$$
\begin{aligned}
& \sigma_{r}=\frac{E}{1-\mu^{2}}\left[\frac{\mu u}{r}+u^{\prime}\right], \\
& \sigma_{\theta}=\frac{E}{1-\mu^{2}}\left[\frac{u}{r}+\mu u^{\prime}\right],
\end{aligned}
$$

where $u$ is the radial displacement, $E$ is the modulus of elasticity, $\sigma_{r}$ and $\sigma_{\theta}$ are radial and circumferential components of the normal stress and $\mu$ is the Poisson's ratio. In the Eqs. (2-3) a prime denotes differentiation with respect to the radius. The disk is symmetric with respect to the mid plane 
and its profiles for thickness and density are assumed to vary as functions of radius (r) (Hojjati \& Jafari, 2007; Hojjati \& Hassani, 2008; Hojjati \& Jafari, 2008; Hojjati \& Jafari, 2009):

$$
\begin{aligned}
& h(r)=h_{\circ}\left(\frac{r}{b}\right)^{-n}, \\
& \rho(r)=\rho_{\circ}\left(\frac{r}{b}\right)^{m},
\end{aligned}
$$

where $n$ and $m$ are geometric parameters $(0 \leq n \leq 1, m \geq 0), b$ is the outer radius of the disk and $h$ 。 is the thickness of the disk at $r=b$. Rotating disks with variable density can be considered as functionally graded materials (FGM) and the literatures devoted to this field is rich (Gamer, 1985; Güven, 1992; Bayat et al., 2008). Although, other material properties such as modulus of elasticity can also be assumed to vary with disk radius, in this research it is arbitrary decided to assume that Young's modulus is constant as the case reported in the previous studies (Gamer, 1985; Güven, 1992; Hojjati \& Jafari, 2008; Hojjati \& Jafari, 2009). Substitution of Eqs. (2-5) in Eq. (1) yields the governing equation for the radial displacement:

$$
\frac{d^{2}}{d r^{2}} u(r)+\frac{(-n+1)}{r} \frac{d}{d r} u(r)-\frac{(n \mu+1)}{r^{2}} u(r)=-\rho_{0}\left(\frac{r}{b}\right)^{m} \omega^{2} r\left(1-\mu^{2}\right) / E .
$$

This is the differential equation governing on rotating disks in elastic form. In this research we have to use the results of the pervious works by the authors (Hojjati \& Jafari, 2008; Hojjati \& Jafari, 2009) for starting the disks optimization. Therefore solution of the elastic non-uniform thickness and density rotating disk (Eq.6) obtained by Homotopy perturbation method is derived as (Hojjati \& Jafari, 2008):

$$
u(r)=c_{1} r^{\left(\frac{n}{2}+\frac{\sqrt{n^{2}+4+4 n \mu}}{2}\right)}+c_{2} r^{\left(\frac{n}{2}-\frac{\sqrt{n^{2}+4+4 n \mu}}{2}\right)}-\frac{\rho_{\circ} \omega^{2} r^{3}\left(\frac{r}{b}\right)^{m}\left(\mu^{2}-1\right)}{E\left(-m^{2}+(n-6) m-8+n \mu+3 n\right)},
$$

where $c_{1}, c_{2}$ are integration constants. The solution must be completed by incorporating a certain set of boundary conditions. Here, free boundary conditions are considered for annular disks. If both surfaces at inner and outer radius are free of any traction, then the boundary conditions are $\sigma_{r}(a)=\sigma_{r}(b)=0$.

\subsection{KKT Conditions}

The necessary conditions for equality constrain that contained the Lagrange Multiplier theorem generally discussed in textbooks on calculus (Rao, 2009; Peressini et al., 1988; Wide et al., 1970). Then, the necessary conditions for the general constrained problem are obtained as an extension of the Lagrange Multiplier theorem. These are known as KKT necessary conditions. All optimum designs must satisfy these conditions. We now examine the problem for this optimization with equality and inequality constraints in the standard form:

$\min f(x)$

subject to

$$
\begin{aligned}
& h_{i}(x)=b_{i} \quad i=1, \cdots, m \\
& g_{j}(x) \leq c_{j} \quad j=1, \cdots, r
\end{aligned}
$$

In these relations $x=\left(x_{1}, \cdots, x_{n}\right)^{\prime}$ is the vector of design variables, $f(x)$ is an object function, $h_{j}(x)=b_{j}$ is an equality constraint and $g_{j}(x) \leq c_{j}$ is inequality constraint, respectively and they are all twice continuously differentiable functions. If design point $\left(x^{*}\right)$ lies on one or more than one 
constraint surface, the associated constraint is called an active constraint. At constrained minimum, only the active constraints are significant. Hence the first- order optimality condition (Eq. (9)) must be applied to inequality constraint (Peressini et al, 1988):

$\nabla f\left(x^{*}\right)+\lambda^{T} \nabla g\left(x^{*}\right)=0^{T}, \quad \lambda \geq 0, \quad \lambda^{T} g=0$.

Those constraints satisfy Eq. (9) are known as active constraint and the necessary conditions are known as KKT conditions as follow (Rao, 2009),

1. $h_{i}\left(x^{*}\right)=0, \quad g_{j}\left(x^{*}\right) \leq 0$.

$2 . \nabla f^{*}+u^{T} \nabla h^{*}+\lambda^{T} \nabla g^{*}=0^{T}$, where $u \neq 0, \quad \lambda \geq 0, \quad \lambda^{T} g=0$.

where $u=\left(u_{1}, \ldots, u_{m}\right)$ is the vector of a Lagrange multiplier for equality constraints and $\lambda=\left(\lambda_{1}, \ldots . \lambda_{r}\right)^{\prime}$ is the vector of a Lagrange multiplier for inequality constraints. Here $x^{*}$, the minimizer, is assumed to be a regular point. A point that satisfies the KKT conditions is called a KKT point and may not be a minimum since the conditions are not sufficient. Second-order information is necessary to verify the nature of a KKT point. The second-order sufficiency conditions are as follow:

If a KKT point $x^{*}$ exists, such that the Hessian of the Lagrangian on the subspace tangent to the active constraints is positive definite at $x^{*}$, then $x^{*}$ is a local constraint minimum. We have defined Hessian matrix as the second partial derivatives of $f(x)$ as follow (Rao, 2009):

$J_{x=x^{*}}=\left[\begin{array}{ll}0 & \nabla g_{j} \\ \nabla g_{j}^{T} & L_{X X}\end{array}\right]_{x=x^{*}} \quad j=j$ for activeconstra int

where,

$L_{x x}=\left[\frac{\partial^{2} L}{\partial x_{i} \partial x_{j}}\right]_{x=x^{*}} \quad i=1 \ldots . n, \quad j=1 \ldots . n$

We summarized these results here for the sake of simplicity,

Model: $\quad \min f \quad$ subject to

$h=0, g \leq 0$

Lagrangian function: $\quad L=f+u^{T} h+\lambda^{T} g$

KKT

conditions:

$\nabla f+u^{T} \nabla h+\lambda^{T} g=o^{T}$.

$h=0, g \leq 0$

$u \neq 0, \lambda \geq 0, \lambda^{T} g=0$

Then for Eq. (8), the Lagrangian function equals to:

$L(x, u, \lambda)=f(x)+\sum_{i=1}^{m} u_{i}\left(h_{i}(x)-b_{i}\right)+\sum_{j=1}^{r} \lambda_{j}\left(g_{j}(x)-c_{j}\right)$

If $\lambda>0$, then $x^{*}$ is a local minimum, 
$\nabla_{x} L\left(x^{*}, u^{*}, \lambda^{*}\right)=\nabla f\left(x^{*}\right)+\sum_{i=1}^{m} u_{i}^{*} \nabla h_{i}\left(x^{*}\right)+\sum_{j=1}^{r} \lambda_{j}^{*} \nabla g_{j}\left(x^{*}\right)=0$

$\lambda_{j}^{*} \geq 0, \quad \lambda_{j}^{*}\left[g_{j}\left(x^{*}\right)-c_{j}\right]=0$

$g_{j}\left(x^{*}\right) \leq c_{j}, j=1, \cdots, r$.

Simultaneous solution of the above equations provides the calculations of $x^{*}$.

\section{Weight optimization of rotating disk}

\subsection{Weight of annular disk}

The weight of disk can be written as weight $=w=\gamma \times V$ where $V$ is volume of annular disk and $\gamma=\rho g$ is the specific gravity of disk where $g$ is the gravity constant.

weight $=w=\gamma^{*} V$

The volume of the body results from rotating the region given in Fig. 2 about vertical axis is:

$V=2 \pi \int_{a}^{b} r(f(r)-g(r)) d r$

where,

$$
\begin{array}{ll}
f(r)=h(r)=h_{\circ}\left(\frac{r}{b}\right)^{-n}, & \\
g(r)=-h(r)=-h_{\circ}\left(\frac{r}{b}\right)^{-n}, & r=0.1, \cdots, 0.6
\end{array}
$$

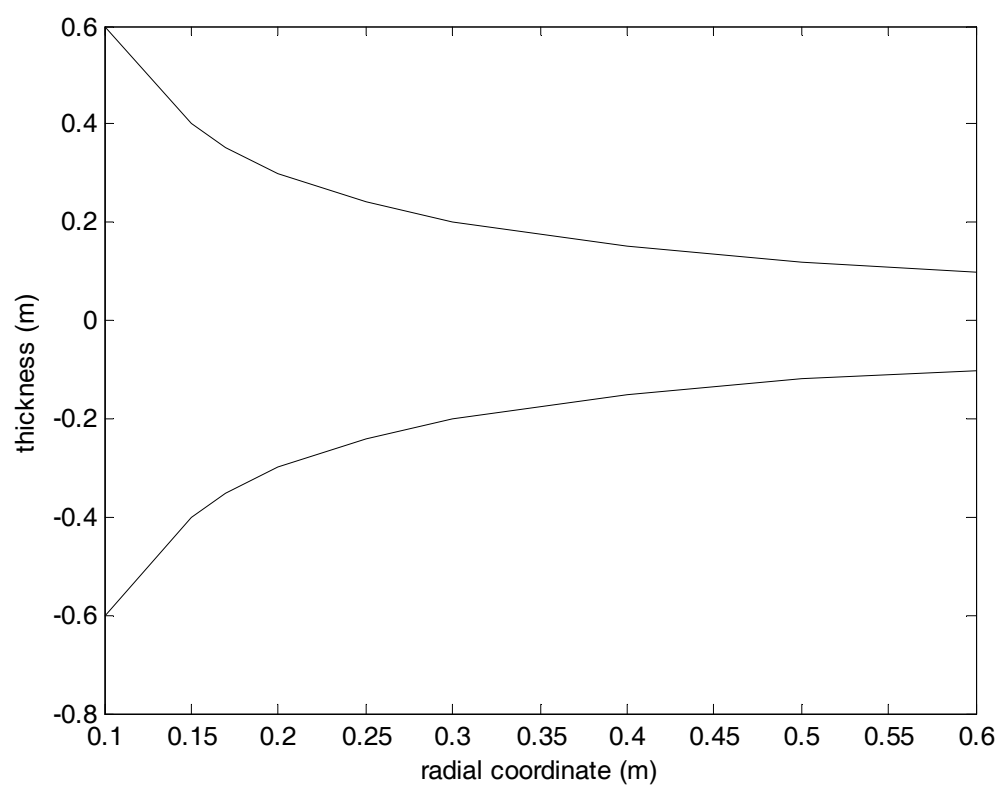

Fig. 2. Disk profile for $n=1, a=0.1 m, b=0.6 m$

Using these relations, the volume of an infinitesimal element can be found as: 
$d V=4 \pi h_{\circ}\left(\frac{r}{b}\right)^{-n}$.

Specific density of disk $\rho(r)$ is assumed to vary with $r$ and it is defined as:

$\rho(r)=\rho \circ\left(\frac{r}{b}\right)^{m}$

where $\rho_{\mathrm{o}}$ is the density at inner radius i.e. $r=b$. Thus, the specific gravity of the disk equals to:

$\gamma(r)=\rho_{\circ} g\left(\frac{r}{b}\right)^{m}$.

By using Eq. (19) and Eq. (21) and setting $g=9.81 \mathrm{~m} / \mathrm{s}^{2}$, the weight of the disk corresponds to:

$$
\begin{aligned}
& w=\int \gamma(r) d V=\int_{r=0.1}^{r=0.6} \rho_{\circ}\left(\frac{r}{b}\right)^{m} g 4 \pi r h \circ\left(\frac{r}{b}\right)^{-n} d r= \\
& -\frac{1}{-m+n-2}\left(\begin{array}{l}
976.22676 \times 1.66667^{-n} \\
\left(-1.166667^{m} \times 10^{-m+n}+36 \times 1.66667^{m} \times 3^{m-n} \times 5^{-m+n}\right)
\end{array}\right)
\end{aligned}
$$

\subsection{Governing constraint of rotating annular disk}

That constrain used in this optimization is that the disk must remain in elastic region based on vonMises criteria. In a plane stress rotating disk von Mises stress corresponds to:

$$
\sigma_{e}=\sqrt{\sigma_{r}^{2}+\sigma_{\theta}^{2}-\sigma_{r} \sigma_{\theta}}
$$

Those stresses components exist in Eq. (23) are evaluated by using Eqs. (2-3) after substitution of Eq. (7) for radial displacement $(u)$. The final relation for von-Mises stress does include the parameters $n$ and $m$ used in Eqs. (4-5).

\subsection{Preparing weight and constraint functions for disk weight minimization}

The optimization problem is reduced to minimization of objective function $W_{\text {disk }}$ subject to the stress constraint, whose complete statement is expressed as,

$\min W_{\text {disk }}$

subject to $\sigma_{e} \leq \sigma_{\text {。 }}$

The differences in scale of the objective function and the constraint may cause some difficulties. Hence, they should be normalized. The weight is normalized by dividing it over the disk at maximum weight conditions i.e. for $m=0$ and $n=1$.

$\bar{W}(n, m)=\frac{W_{d i s k}}{W_{n=1, m=0}}$

Equivalent von Mises stress can be dimensionless by dividing it by yield strength of the material:

$\overline{\sigma_{e}}(n, m, r)=\frac{\sigma_{e}}{\sigma_{\circ}}$ 
From Fig. 3, it is obvious that for different values of $m$ and $n$ parameters, von Mises stress has its maximum value at inner radius i,e. at $r=0.1 \mathrm{~m}$ in our case study. Therefore, the Eq. (25) can be reduced to the following equation which has no terms of $r$ :

$\overline{\sigma_{e}}(n, m)=\frac{\left(\sigma_{e}\right)_{r=0.1}}{\sigma_{。}}$

The angular velocity of rotating disk can also be normalized as (Hojjati \& Jafari, 2009):

$\Omega=\sqrt{\frac{\rho \omega^{2} b^{2}}{\sigma_{\circ}}}$

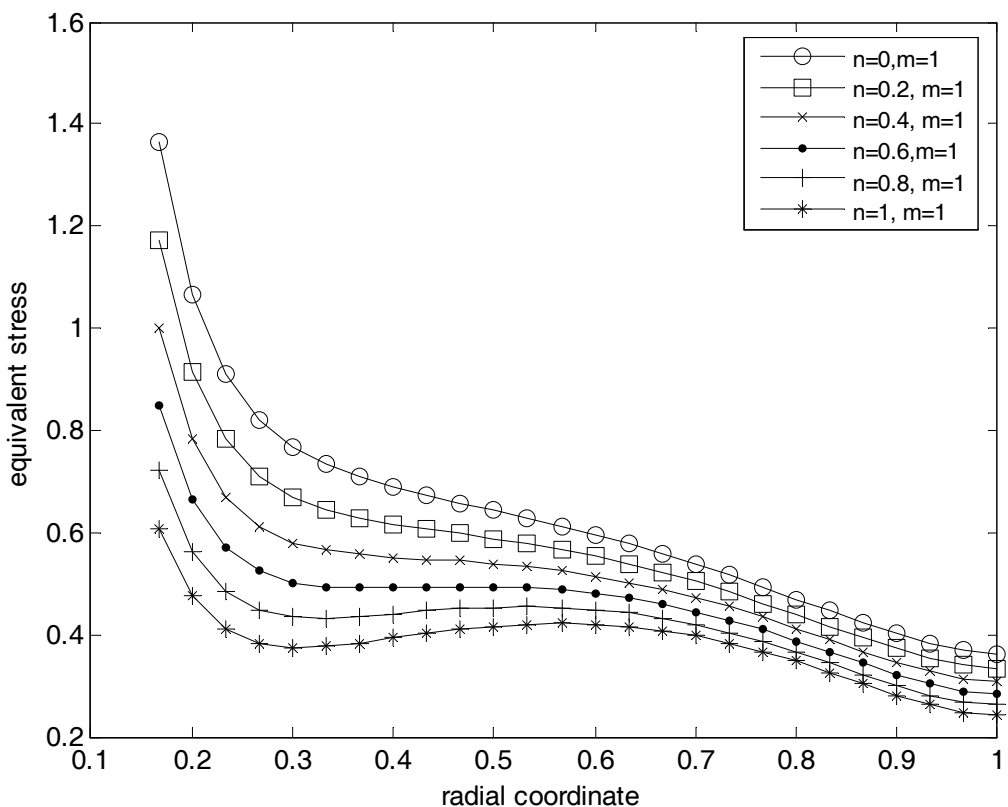

Fig. 3. Equivalent von Mises stresses on annular rotating disk with variable thickness and density for free boundary condition ( $\mathrm{m}=1$ and different value of $n$ at 1.5346 normalizd angular velocity)

\section{Case Study}

To show the performance of KKT optimization method in weight minimization of a rotating disk with variable thickness and density, application of the proposed method is presented here for two cases. The first case is when $\sigma_{e} \leq \sigma_{\circ}$, is assumed as the constraint and the other case is when $\sigma_{e} \leq 0.8 \sigma_{\circ}$, is used as failure stress criterion. The later means that we wish to include a safety factor in the design, for instance, making the maximum von Mises stress as 0.8 times of yield strength of the material. Table (1) shows the material and the geometry properties used in the numerical examples (Jafari \& Hojjati, 2011).

\section{Table 1}

Geometry and material properties of rotating disks

\begin{tabular}{llllllll}
\hline $\mathrm{b}(\mathrm{m})$ & $\mathrm{a}(\mathrm{m})$ & $h_{\circ}(\mathrm{m})$ & $\rho_{\circ}\left(\mathrm{kg} / \mathrm{m}^{3}\right)$ & $E(\mathrm{GPa})$ & $v$ & $\omega(\mathrm{rad} / \mathrm{s})$ & $\sigma_{\circ}(\mathrm{MPa})$ \\
\hline 0.6 & 0.1 & 0.1 & 7850 & 207 & 0.3 & 500 & 300 \\
\hline
\end{tabular}

The optimization problem for the two constraints in normalized form state as follow:

$\min \bar{W}_{\text {disk }}$

subject to $\overline{\sigma_{e}}-A \leq 0$, 
where $A$ is a real number that has value of 1 for the first constraint as $\sigma_{e} \leq \sigma_{\circ}$ and 0.8 for second constraint as $\sigma_{e} \leq 0.8 \sigma$ 。 in normalized form (Jafari \& Hojjati, 2011).

The optimization problem with KKT conditions is formulated as,

minimize $\quad \bar{W}(n, m)$

subject to $\quad \overline{\sigma_{e}}(n, m) \leq A$

Lagrangianfunction:

$L(n, m, \lambda)=\bar{W}+\lambda\left(\overline{\sigma_{e}}-A\right)$

KKT conditions:

$\nabla \bar{W}+\lambda \nabla\left(\overline{\sigma_{e}}-A\right)=0$

$\lambda \geq 0, \quad \lambda\left(\overline{\sigma_{e}}-A\right)=0$

These relations can now be summarized as:

$\frac{\delta \bar{W}}{\delta n}=0, \quad \frac{\delta \bar{W}}{\delta m}=0, \quad \frac{\delta \bar{W}}{\delta \lambda}=0, \lambda \geq 0, \lambda\left(\overline{\sigma_{e}}-A\right)=0, \quad A=1$ or 0.8

Table 2 presents the results for the first constraint case while Table 3 shows the same results for the second constraint case, i.e. $A=1$. All the presented results are for $\Omega=1.5346$.

Table 2

The optimal results for disk weight optimization under $\sigma_{e} \leq \sigma_{\text {。 }}$ constraint with A=1

\begin{tabular}{lllll}
\hline & Value of $n$ & Value of $m$ & Lagrange multiplier & Weight reduction \\
\hline KKT & 0.43069 & 0.88913 & 0.25942 & $68 \%$ \\
\hline
\end{tabular}

Table 3

The optimal results for disk weight optimization under $\sigma_{e} \leq 0.8 \sigma_{\circ}$ constraint with $\mathrm{A}=0.8$

\begin{tabular}{lllll}
\hline & Value of $\mathrm{n}$ & Value of $\mathrm{m}$ & Lagrange multiplier & Weight reduction \\
\hline KKT & 0.73873 & 0.85014 & 0.43020 & $61 \%$ \\
\hline
\end{tabular}

The values of $\lambda$ for both cases are positive, which confirm that the calculated optimum point gives the minimum weight of disk. Based on Eq. (11), one can realize that the Hessian matrix for the above values is positive definite. Therefore, the sufficient condition is satisfied and these values minimize weight of the disk for given constraint.

\section{Discussions}

As it was mentioned before, KKT optimization method has been used for weight minimization of a rotating disk with non uniform thickness and material properties. For the first case of constraint defined as $\sigma_{e} \leq \sigma_{\circ}$, Fig. 4 shows the disc profile before $(n=1)$ and after optimization. Using the optimization method reviewed here, after design/optimization process, a weight reduction of $68 \%$ and maximum von Mises stress of $300 \mathrm{MPa}$ (equal to the yield stress) was achieved. In Fig. 5 von Mises stress distribution for different radii has been shown for the optimized values of $m$ and $n$. The obtained disk profile for the second case of constraint defined by $\sigma_{e} \leq 0.8 \sigma_{\circ}$ is shown in Fig. 6 . Using the optimization method, a weight reduction of $61 \%$ was achieved. In Fig. 7 von Mises stress distributions across the disk thickness for above calculated $n, m$ values have been presented. It can be 
seen that the von Mises stress throughout the disk thickness has been kept under the desired value as expected.

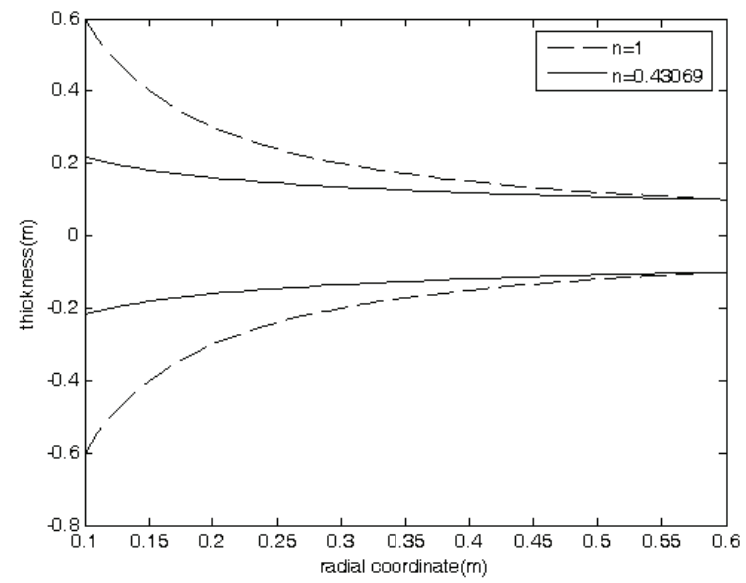

Fig. 4. Thickness profilefor $\mathrm{n}=1$ and $\mathrm{n}=0.43069$ under $\sigma_{e} \leq \sigma_{\text {。 }}$ constraint

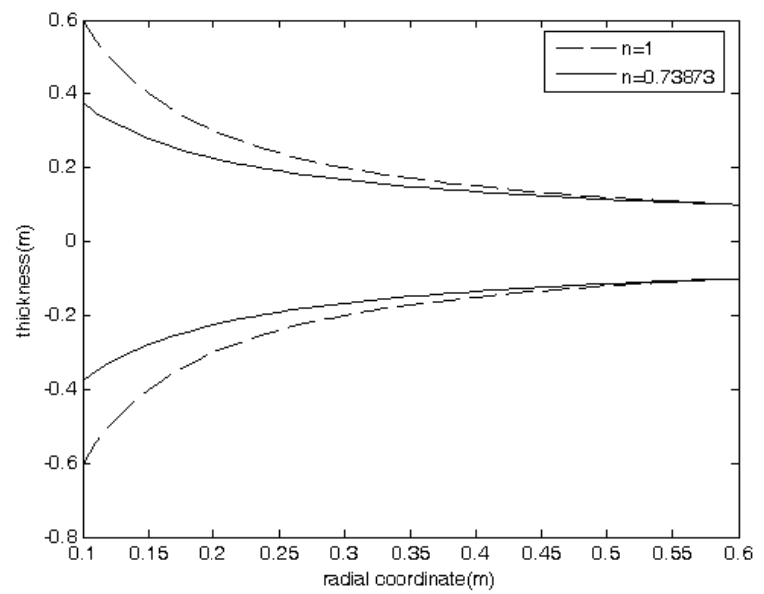

Fig. 6. Thickness profile for $\mathrm{n}=1$ and $\mathrm{n}=0.73873$ under $\sigma_{e} \leq 0.8 \sigma_{\text {。 }}$ constraint

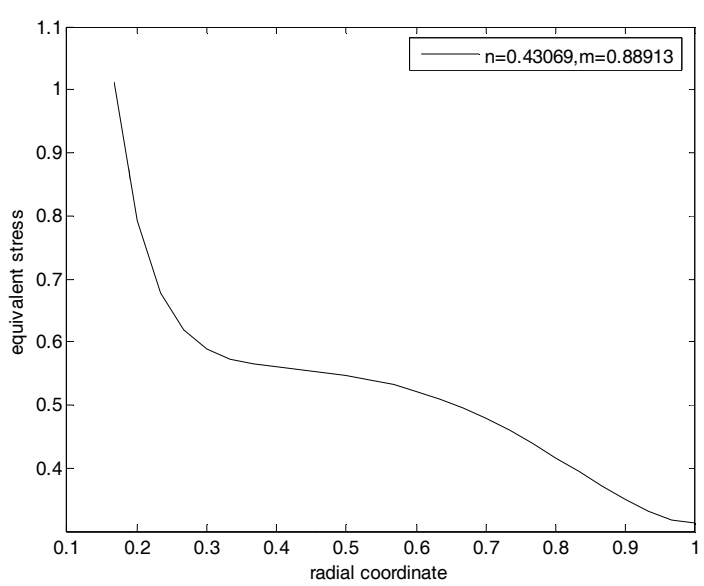

Fig. 5. von Mises stress for $n=0.43069$ and $\mathrm{m}=0.88913$ at 1.5346 normalizd angular velocity under $\sigma_{e} \leq \sigma_{\text {。 }}$ constraint

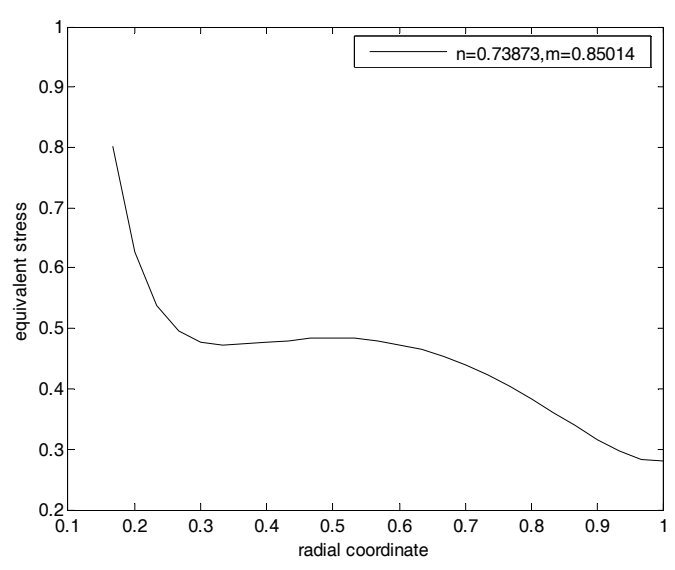

Fig. 7. von Mises stress for $\mathrm{n}=0.73873$ and $\mathrm{m}=0.85014$ at 1.5346 normalizd angular velocity under $\sigma_{e} \leq 0.8 \sigma_{\text {。 }}$ constraint

\section{Concluding remarks}

In this paper, rotating annular disks of variable thickness and density have been optimized for minimum weight subject to fulfillment of certain condition on the value of the maximum von Mises stress. The Lagrange multiplier along with KKT conditions theorem have been used in optimization. A plane stress condition has been assumed. Semi exact solution of the disk previously developed by the authors has been used to evaluate the magnitude of the stress components of the disk. The geometrical parameters of the shape of the profile of a given disk with predefined inner and outer radii have been found which reduces the weight of the disk by as much as $68 \%$ while the condition on the maximum von Mises stress is satisfied. 


\section{References}

Bayat, M, Saleem. M, Sahari. B.B, Hamouda, A.M.S, Mahdi, E. (2008). Analysis of functionally graded rotating disks with variable thickness. Mechanic Research Communications, 35, 283-309.

Cheu, T. (1990). Procedures for shape optimization of gas turbine. Journal of computer structural, $54,1-4$.

Fox, RL. (1970). Optimization methods for engineering design. London: Addison-Wesley.

Gramer, U. (1983). Tresca's yield condition and the rotating solid disk. Journal of Applied Mechanical Engineering, 50(3), 676-678.

Gramer, U. (1984). The elastic-plastic stress distribution in the rotating annulus and in the annulus under external pressure. Journal of Applied Mathematics \& Mechanics/Zeitschrift, 64, 126-128.

Gramer, U. (1985). Distribution in the rotating elastic-plastic disk. Journal of Applied Mathematics \& Mechanics/Zeitschrift, 65, 136-137.

Güven, U. (1992). Elastic-plastic stresses in a rotating annular disk of variable thickness and variable density. International Journal of Mechanical Science, 43, 1137-1153.

Güven, U. (1994). The fully plastic rotating disk of variable thickness. Journal of Applied Mathematics \& Mechanics/Zeitschrift, 74, 61-65.

Güven, U. (1995). On the applicability of Tresca's yield condition to the linear hardening rotating solid disk of variable thickness. Journal of Applied Mathematics \& Mechanics/Zeitschrift, 75, 397-398.

Hojjati, M. H, \& Jafari, S. (2007). Variational iteration solution of elastic non uniform thickness and density rotating disks, Far East Journal of Applied Mathematics, 29, 185-200.

Hojjati, M. H, \& Hassani, A. (2008). Theoretical and numerical analyses of rotating discs of nonuniform thickness and density. International Journal of Pressure Vessels and Piping, 85, 694700.

Hojjati, M. H, \& Jafari, S. (2008). Semi Exact Solution of Elastic Non Uniform Thickness and Density Rotating Disks by Homotopy Perturbation and Adomian's Decomposition Methods Part I: Elastic Solution. International Journal of Pressure Vessels and Piping, 85, 871-878.

Hojjati, M. H, \& Jafari, S. (2009). Semi- Exact solution of non uniform thickness and density rotating disks Part II: Elastic-strain hardening solution. International Journal of Pressure Vessels and Piping, 86, 307-318.

Jafari, S, \& Hojjati, M. H. (2011). Modern optimization methods in minimum weight design of elastic annular rotating disk with variable thickness. Advances in Applied Physics \& Material Science-APMAS, 2011.

Malkov, V. P., \& Salgankays, E. A. (1976). Optimum material distribution in rotating disks for minimum strength. Sov Aeronaut, 19, 46-50.

Rao, S. (2009). Engineering Optimization: Theory and Practice, $4^{\text {th }}$ Edition, Wiley \& Sons.

Peressini, AL, Sullivan, FE. (1988). The Mathematics of Nonlinear Programming, Berlin, New York, Springer.

Timoshenko, S. \& Goodier, J, N. (1970). Theory of Elasticity, 3rd edition. McGraw-Hill, New York.

Vanderplaats, G. N. (1990). Numerical optimization techniques for engineering design with applications. 2nd ed. New York: McGraw-Hill.

Wide, D.J., \& Papalambros, P. Y. (2000). Principles of optimal design, modeling and computation, $2^{\text {nd }}$ edition, Cambridge University Press.

Zeinkiewics, OC, Campbell, JS. (1973). Shape optimization and sequential linear programming in optimum structural design. New York: Wiley. 